\title{
Streamlining clinical practice in an Australian community rehabilitation service using The Calderdale Framework
}

\author{
Sarah A Patterson, Tracy A Comans, Erin L Pitt, Michelle L Currin
}

Background/Aims: With increased budgetary demands, pressure exists to create a flexible workforce and ensure efficient service delivery within health care services. This study investigated the impact of using The Calderdale Framework on team dynamics, client focus and communication, and resource use in an existing community rehabilitation service.

Methods: The Calderdale Framework provided a structured process where clinicians were able to determine relevant tasks that could be shared with other health professionals to develop a streamlined transprofessional screening tool. Associated risks were reviewed and the resulting training and governance structures were embedded into practice. Staff views and resource usage costs were collected at three time points during the implementation process.

Findings: Qualitative data from staff suggest a positive impact of The Calderdale Framework on team dynamics, understanding each other's roles, and client focus. Resource usage analysis revealed an increase in the number of client services provided, as well a reduction in cost per client appointment. Conclusions: This study provides further insight into the processes, resource usage outcomes and staff reflections of using The Calderdale Framework. This structured tool appears beneficial to develop a flexible workforce and an efficient service within this community rehabilitation context.

Key words: The Calderdale Framework $\square$ Transprofessional practice $\square$ Resource use $\square$ Community rehabilitation

Submitted 15 June 2015; sent back for revisions 10 July 2015; accepted for publication following double-blind

Sarah A Patterson, Advanced occupational therapist, Community Adult Rehabilitation

Service, Metro South Hospital and Health

Service, Australia;

Tracy A Comans,

Principal research fellow, Menzies Health Institute

Queensland, Griffith

University and Metro North Hospital and Health

Service, Australia;

Erin L Pitt,

PhD student, Menzies Health Institute

Queensland, Griffith

University, Australia;

Michelle L Currin;

Programme coordinator,

Community Adult

Rehabilitation Service, Metro South Hospital and Health Service, Australia.

Correspondence to: Sarah A Patterson E-mail:

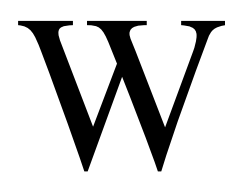

ith increasing health demands and workforce shortages, traditional allied health work roles and models of care are unlikely to sustain our changing health care needs (Smith and Duffy, 2010). The typical allied health workforce is comprised of formalised professional specific groups who work within a pre-determined skill set. This model of care is underpinned by the assumption that staff can only perform tasks effectively and safely from their professionspecific skill base, and clients are therefore required to see multiple clinicians to achieve their desired outcomes (Nancarrow, 2015).

To assist with service demand and build workforce capacity, there is a shift toward developing a skilled flexible workforce, which focuses on client need rather than traditional professional work roles (Nancarrow, 2004). Evidence is emerging that care can be provided by more than one clinician (Booth and Hewison, 2002) through delegation of tasks to therapy assistant staff (Nancarrow et al, 2012) and sharing job tasks between professionals (i.e. transprofessional practices) (Gilmore et al, 2011). Although delegation to therapy assistants is established in some health care settings (Smith and Duffy, 2010) and preliminary research into advanced therapy assistants appears favourable (Wood et al, 2011), further development and research into streamlining flexible workforces is warranted. Currently, there is limited evidence in Australia regarding the effectiveness and sustainability of initiatives regarding role extension through transprofessional practices (Gilmore et al, 2011).

Community rehabilitation is an area of allied health care that is in particular demand, especially considering Australia's ageing population and rising incidence of chronic disease (Duckett, 2005). Community rehabilitation is recognised as an integral part of Australian health care (Kendall et al, 2009), with available literature suggesting that such services are able to achieve equivalent functional outcomes when compared with traditional hospital-based services (Crotty et al, 2008; Brusco et al, 2014), with lower costs (Brusco et al, 2014) and reduced therapy hours (Crotty et al, 2008). Service demand is 
necessitating that community rehabilitation services explore flexible workforce options and redesign of their model of care.

Workforce analysis and redesign tools, such as The Calderdale Framework (Smith and Duffy, 2010), have been developed to assist with risk minimisation of role sharing as flexible workforces are created. The Calderdale Framework was developed to provide a systematic method of reviewing health service skill mix in order to devise new roles and new ways of working for streamlining services and providing effective client-centred care. As shown in Figure 1, the framework is comprised of seven stages to promote successful changes in practice, with emphasis on clinicians driving changes within their clinical settings (Smith and Duffy, 2010). The process involves analysing tasks carried out in health service teams to identify the range of tasks completed by health practitioners.

Using this systematic approach, it is determined by consensus which tasks must remain uni-professional, which tasks could potentially be shared across professions to become transprofessional practices, and those that can be delegated to the support/assistant workforce. This process leads to the development of detailed competencies, based on tasks that are needed to deliver client-focused services. Subsequently, this provides the basis for training of staff, giving them a record of competence and ensuring they are fit for purpose in their new role.

In this study, The Calderdale Framework was implemented in a community rehabilitation team to streamline clinical practice by minimising duplication of roles and more effectively utilising multi-skilled staff through the development of a transprofessional holistic screening tool. It was hypothesised that using The Calderdale Framework would enhance team dynamics, client focus and communication. It was also hypothesised that this transprofessional tool would make more efficient use of clinicians' time through more effective screening and streamlining processes for interventions, and that clients who accessed the service would benefit from having a more coordinated care plan.

\section{AIMS}

The aim of this study was to investigate the impact of using The Calderdale Framework on team dynamics, client focus and communication. A secondary aim was to assess the impact of the framework on clinician time, client time and other resource use within the team.

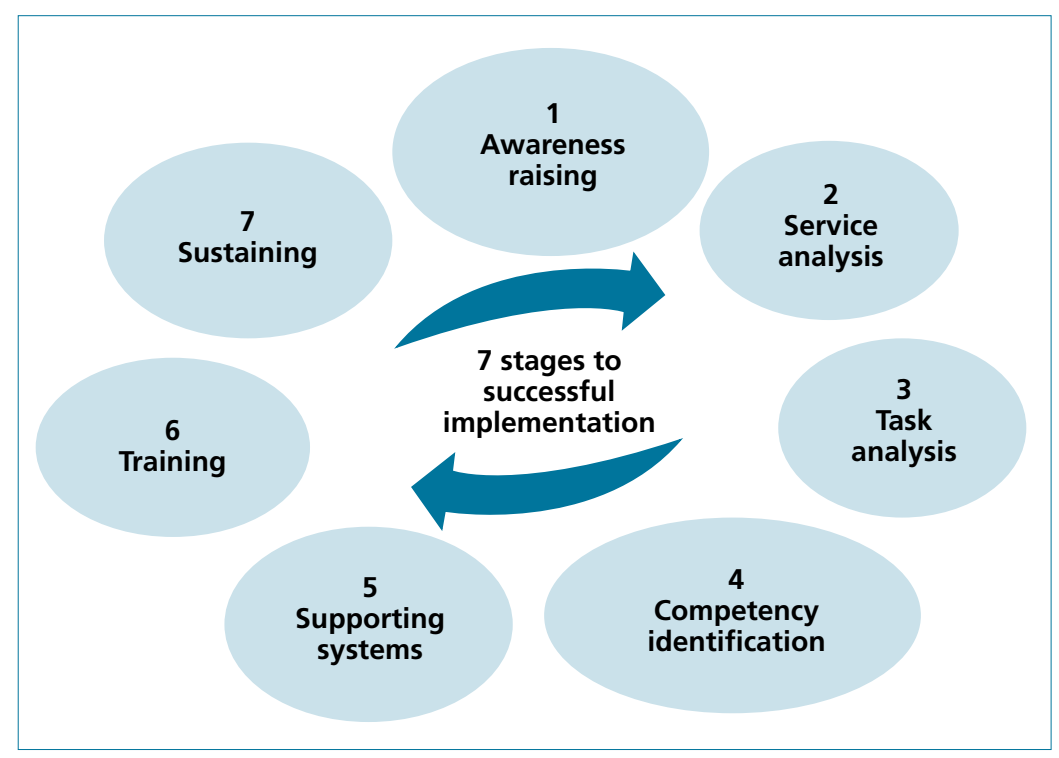

Figure 1. The Calderdale Framework. Reproduced with permission

\section{METHODS}

This study used a pre-post design with a combination of quantitative and qualitative data collection techniques, such as regular monitoring of resource usage data and staff focus groups. The effect of the service delivery change associated with The Calderdale Framework was reviewed by examining resource usage data. The qualitative groups were facilitated to explore staff perceptions of the change process and the associated effect on client care, team dynamics and communication.

\section{Settings and timescales}

The community rehabilitation service was located in Queensland, Australia and covered a large geographical area in a predominately urban area, with four subteams across the service delivery area. The service comprised 23 full-time equivalent allied health staff across the disciplines of physiotherapy, occupational therapy, speech pathology, dietetics and social work. Traditional multidisciplinary community rehabilitation services were already well established within the Metro South Hospital and Health Service District prior to this transprofessional practice redesign implementation and research. Utilisation of The Calderdale Framework commenced in March 2012 and was finalised in March 2013.

Baseline measures of the staff focus groups were collected in March 2012, with repeat measures collected in October 2012 and in April 2013. Information on resource use and staff time was extracted from routinely collected administrative data in April 2012, October 2012 and April 2013. 


\section{Participants}

All full-time, part-time and locum staff working within the community rehabilitation service during the study time period were invited to participate in the 30-minute staff focus groups. Thirty allied health staff from physiotherapy, occupational therapy, speech pathology, dietetics and social work participated in each of the focus group sessions.

Resource usage information was extracted from new client data, those currently waiting for services, as well as clients already actively receiving interventions. Data from only one of the subteams were utilised for this study, as data from other subteams were confounded during the research period due to unrelated factors such as merging client databases, staff changes and altering clinical focus. The subteam utilised in this study maintained a constant staffing level of 3.8 full-time equivalent over the research period and was not subject to any other external factors. It is therefore hypothesised that any changes noted in this dataset would be related to the introduction of transprofessional practices.

\section{Ethical approval}

This study was granted approval by the Princess Alexandra Hospital Human Research Ethics Committee (HREC/11/QPAH/542) in accordance with the Australian National Health and Medical Research Council Guidelines. Written consent was obtained from all staff participating in the focus groups, with all research material kept in appropriate, secured locations.

\section{Interventions}

The Calderdale Framework was used to develop a transprofessional screening tool that would be used by all clinicians to gather initial information from clients about their rehabilitation needs and goals. A steering committee was organised to oversee the project and consisted of the project officer and representatives from the key stakeholders, including the project sponsor, programme manager and allied health representation from the associated hospitals. A working party of senior clinicians within the community rehabilitation teams was also established to assist with implementing the changes at a clinical level.

The initial focus of The Calderdale Framework was to ensure managers and clinicians were aware of and engaged in the process. Thus, the key stakeholders were initially identified and communication plans detailing the contextspecific use of the framework were established. A monthly newsletter was established to send to all key stakeholders to provide project updates and open lines of communication.

A pre-implementation questionnaire was used to gather background information on the agreed review topics prior to the service analysis discussions. Service analysis was completed with the senior clinical staff to identify and clarify current service functions in each of the professions. This reviewed types of clients, referral source and a typical client journey through the service, including assessments, screenings (both informal and standardised) and interventions. A spreadsheet was created with all the data to highlight the similarities, differences and potential duplications of services and was re-distributed for further comment. Core screening items were identified from this information (i.e. screens currently completed by all professions), as well as specific professional screening items required for further task analysis.

During task analysis, each of the professionspecific screening tasks were evaluated to determine whether they could be clinically shared, modified to allow them to be clinically shared or if they were required to remain uni-professional. If current risks prevented the task from being shared in its usual form, discussions were also held as to how the task might be modified to allow sharing if possible. As part of task analysis, consideration was given to the use of these screening tasks as part of the transprofessional screening tool. Thus, each professional group was required to share a screening tool to be included in the transprofessional screen to assist with client care plans and aid in decision making as to whether a referral to a particular profession was necessary.

The task analysis meetings enabled the profession-specific screening tasks to be identified and, subsequently, the transprofessional screening tool was developed. The tool combined both the core screening tasks (i.e. those tasks already completed by all professions, with no additional training needs identified) as well as professionspecific screening tasks, which formed the basis of the competencies and training.

Under The Calderdale Framework, competencies provide details of the performance criteria required to perform each of the tasks, as well as a record of the trainee's ability to undertake the tasks required. The project officer initiated the competency writing by investigating other Calderdale Framework competencies already in existence and modifying these as required for the local context. Senior staff from the various professions within the working party were responsible for providing competency training to other health professionals. 
To ensure that the community rehabilitation service was equipped to manage the new roles, service delivery and scope of practice, the project officer implemented a number of strategies to support these new changes. Information technology systems were updated to reflect the new transprofessional practices, including referral pathways, documentation and electronic client charts. An additional monthly in-service was established for the team members to provide a set time for transprofessional learning and reflective practices. Furthermore, case conference procedures were refined to promote further transprofessional discussions and reflections within a structured process.

In order to sustain the efforts and changes made through The Calderdale Framework, a number of strategies were established, namely: i) an orientation package and checklist were developed to ensure all new and rotational staff as well as students were aware of the practices within the service and how the competencies were derived, and ii) workplace instructions related to competencies and training procedures were created and implemented.

\section{Outcome measures}

Qualitative data associated with the new transprofessional screening tool were derived through staff focus groups. All staff working in the service within the research time frames were invited to attend. The focus groups were facilitated during work time by an independent workforce development officer and explored staff perceptions of the new screening tool, as well as their perceived confidence to undertake their current and new roles. Staff were asked: 'What should we start?', 'What should we stop?', and 'What should we continue?'. Discussions were summarised and recorded by the workforce development officer running the focus groups.

For resource usage, information was extracted from routinely collected administrative data at the three time points (initial, mid-way and final) for periods of 30 days each. Demographic details were collected, including age, gender and presenting condition, which was categorised as stroke, falls and ageing, Parkinson's disease or others (acquired brain injury, neurological and general medicine issues). Details of each appointment were recorded, including date, duration, location (home or community health) and mode (in person, telephone, written or indirect).

Individual therapist information was collected for each appointment, including location of the therapist's clinic base, their profession and Queensland Health Practitioner salary award level. Travel time and distance for clients and staff were calculated by estimating both distance and time from the client's suburb to the appointment location (when the appointment was in person and other than a home visit) using freely available mapping data. Total client time was calculated by summing total travel time and the appointment duration. This was similarly calculated for clinicians' total time.

Each clinician's cost per minute was generated based on the highest band of the clinician's salary award level. Vehicle costs were established using a rate of AUD \$0.74 per kilometre travelled based on Australian Taxation Office figures. Total therapist travel time was generated by summing the appointment duration and total clinician travel time. The cost of the therapist's travel time was generated by summing the vehicle cost and total therapist travel time. Cost of therapist was generated by summing the duration of appointment and total therapist travel time, and multiplying this by the therapist's cost per minute. The total cost per appointment was generated by summing the cost of therapist, cost of therapist's travel time and vehicle cost.

\section{Data analysis}

Analysis of the focus groups was completed by the project officer and another experienced staff member in the community rehabilitation team who was not able to attend the focus groups. Analysis involved two components where the transcripts were read and re-read independently to identify key thoughts and ideas within each of the focus groups at the various time points. Following this, each analyser then developed overall key concepts across the focus groups before finally meeting to collaborate, refine and define final themes. The key thoughts and ideas were grouped into themes and arranged by the analysers to determine theme associations and develop a framework to explain the findings.

Descriptive statistics were used to describe resource use, including average cost per client and average cost per appointment at each of the three time points. Statistical analysis was performed using Stata version 12.0.

\section{FINDINGS}

\section{Qualitative}

Thematic analysis of the initial, mid-way and final focus groups indicated three general themes:

client-focused service

Team dynamics

Communication and change management. 


\section{Client-focused service}

This theme was consistent across both initial and mid-way focus groups, with staff expressing a strong desire to continue and develop services that are client-focused. This also included ensuring that clients are accommodated during any transitions. At the mid-way focus group, staff commented that the new approach allowed the service to be about client needs, rather than individual professional service needs. During the final focus group, staff discussed improvements to client care as part of the service change. Comments received from staff included:

The need to maintain a client focus throughout service changes

The service is becoming more client-focused rather than professional-focused.

\section{Team dynamics}

Team dynamics was a noticeable theme that emerged during the mid-way and final focus groups, with staff reporting improvements in relation to team cohesion, understanding of professions and increased communication between allied health professionals. During the final focus group, staff reported they felt the team was supportive of each other, with greater sharing of workloads noted and a reduction in single-discipline interventions. It was also reported that case conference meetings were a great forum for enabling team discussions;

Table 1. Client demographic and appointment details across three time points

\begin{tabular}{|c|c|c|c|c|}
\hline & & Time point & & \\
\hline & Initial & Mid-way & Final & Total \\
\hline Demographic characteris & & & & \\
\hline Number of participants & 25 & 33 & 61 & 119 \\
\hline Mean (SD) age (years) & $73(11.5)$ & $75(11.3)$ & $68.6(15.5)$ & $71.3(13.9)$ \\
\hline Age range (years) & $43-89$ & 44-94 & 24-94 & 24-94 \\
\hline Gender (female) & $15(60 \%)$ & $15(45 \%)$ & $34(56 \%)$ & $64(54 \%)$ \\
\hline $\begin{array}{l}\text { Presenting condition } \\
\text { Stroke } \\
\text { Falls and ageing } \\
\text { Parkinson's disease } \\
\text { Other* }\end{array}$ & $\begin{array}{l}4(16 \%) \\
8(32 \%) \\
7(28 \%) \\
6(24 \%)\end{array}$ & $\begin{array}{l}4(12 \%) \\
17(52 \%) \\
11(33 \%) \\
1(3 \%)\end{array}$ & $\begin{array}{l}15(25 \%) \\
20(33 \%) \\
18(30 \%) \\
8(13 \%)\end{array}$ & $\begin{array}{l}23(19 \%) \\
45(38 \%) \\
36(30 \%) \\
15(13 \%)\end{array}$ \\
\hline Appointments & & & & \\
\hline Number of participants & 64 & 125 & 196 & 385 \\
\hline $\begin{array}{l}\text { Location } \\
\text { Home setting } \\
\text { Community service }\end{array}$ & $\begin{array}{l}40(63 \%) \\
24(38 \%)\end{array}$ & $\begin{array}{l}59(47 \%) \\
66(53 \%)\end{array}$ & $\begin{array}{l}81(41 \%) \\
115(59 \%)\end{array}$ & $\begin{array}{l}180(47 \%) \\
205(53 \%)\end{array}$ \\
\hline $\begin{array}{l}\text { Mode of appointment } \\
\text { In person } \\
\text { Telephone } \\
\text { Written } \\
\text { Indirect }\end{array}$ & $\begin{array}{l}56(89 \%) \\
4(6 \%) \\
2(3 \%) \\
1(2 \%)\end{array}$ & $\begin{array}{l}66(53 \%) \\
25(20 \%) \\
4(3 \%) \\
30(24 \%)\end{array}$ & $\begin{array}{l}108(55 \%) \\
49(25 \%) \\
25(13 \%) \\
14(7 \%)\end{array}$ & $\begin{array}{l}230(60 \%) \\
78(20 \%) \\
31(8 \%) \\
45(12 \%)\end{array}$ \\
\hline
\end{tabular}

however, staff acknowledged that improvements could still be made in the coordination of client care for part-time staff not able to attend this forum. Summary comments from staff included:

There is improved and increased communication between disciplines

Staff are given the opportunity to learn about what other professions do

The changes enable sharing of workloads and create a sense of team spirit.

\section{Communication and change management}

Management of the change processes within The Calderdale Framework was another theme that emerged from all focus group discussions. In all three focus groups, the staff requested increased clear communications and information about the changes. During the initial focus group, staff indicated a desire for additional information as to how the new screening tool would be developed, while during the mid-way focus group, information needs shifted more towards how to carry out the new changes proposed. During the final focus group, staff continued to express concerns regarding clarity of information and the way it was communicated.

Staff also expressed, during the mid-way and final focus groups, a need to be supported during the time of change, with consideration given to the frustrations of operational issues associated with the change process. This included concerns about paperwork duplication, confusion with the new systems of screening clients, and the dedication of staff to the new systems. Comments received from staff included:

The need for a definite plan about where services are going

Not to overwhelm staff with paperwork, as forms are changing all the time

The need to improve communication between managers and the team, with a focus on better explanations of decision and plans

The need to allow staff more time to learn changes and new systems of working.

\section{Quantitative}

Demographic and appointment details are shown in Table 1. A total of 119 clients were seen over the three time periods. There were slightly more females than males in the sample. Most participants presented for stroke, Parkinson's disease or issues related to falls and ageing. The number of clients seen increased over the three time points. The location of appointment changed over the course of the year, moving from a predominately home-based setting to a centre-based, community health setting. 
The cost per appointment over the three time periods is presented in Figure 2. The average cost of a single appointment dropped between the pre- and post-implementation periods. The total cost per client remained fairly consistent over the three time periods, with a mean \pm SD of AUD \$235 \pm 247 , AUD \$254 \pm 249 and AUD \$216 \pm 223 for time periods 1, 2 and 3, respectively, as clients received an increased number of appointments.

\section{DISCUSSION}

The purpose of this review was to explore the use of The Calderdale Framework in developing a transprofessional screening tool, associated service changes and staff training. The impact of this service delivery change was evaluated qualitatively via staff focus groups to explore staff perceptions of the process. This study also reviewed the resource usage costs associated with the change.

Overall, the introduction of The Calderdale Framework appeared to have a positive impact on increasing efficiency in the community rehabilitation service measured. This included both increasing the number of services within a fixed budget constraint and also reducing the cost per service item. Furthermore, implementation of the framework appears to be favourable with clinicians, who reported improved team dynamics, understanding of each other's role and improving client focus associated with The Calderdale Framework processes and changes. Consequently, it is believed that this framework provides a useful structure to enable costeffective, transprofessional practices among allied health professionals. Figure 3 shows the potential relationship between these findings.

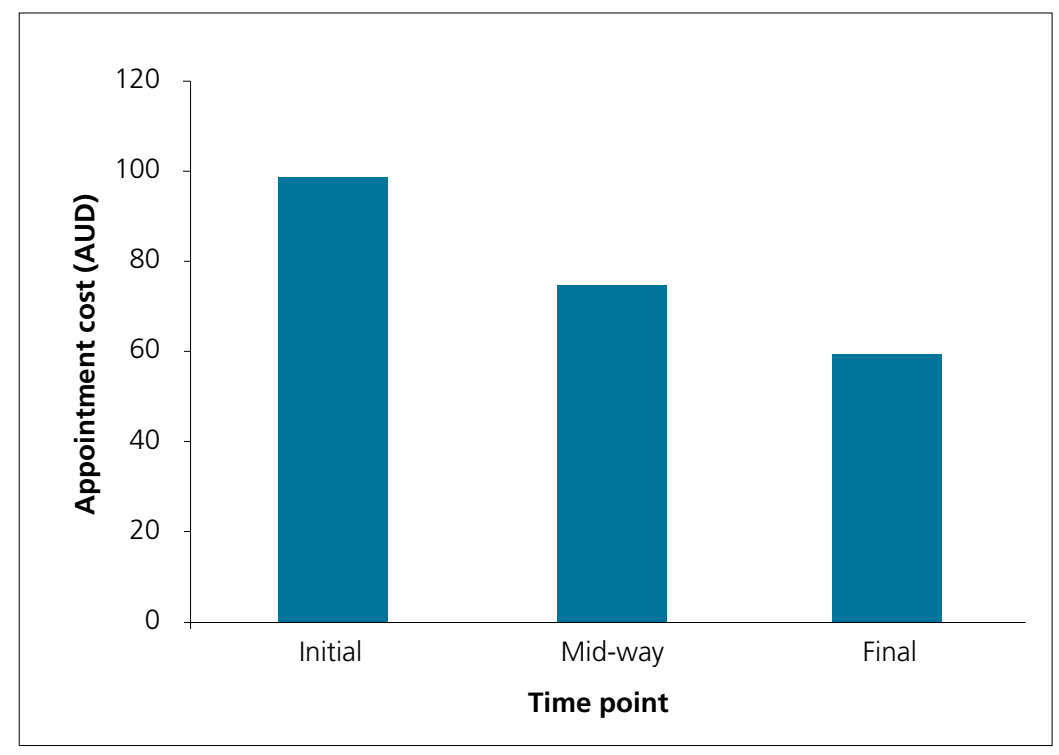

Figure 2. Mean cost per appointment

While transprofessional practices may have the potential to reduce health care demands, it has been suggested that initiatives to date have not been consistent, resulting in subsequent evidence gaps (Gilmore et al, 2011). As such, The Calderdale Framework may provide a consistent, clear template to guide, manage and evaluate such service changes. Used widely in the UK, research around The Calderdale Framework is beginning to emerge in Australia (Nancarrow et al, 2012).

Negotiating change and embedding any new clinical practices is a complex process. The Queensland Government recognises that staff capacity to participate in change is influenced by their inclusion in the process and understanding of the benefits of the change (Queensland Government, 2009). These core change management principles underpin The Calderdale Framework change management process, with early steps to engage key stakeholders and align



Figure 3. Flow diagram of the changes associated with the implementation of The Calderdale Framework 
the workforce with the development of the screening tool. This is driven by clinical staff by relating to their needs in their local context. Despite this, reflections from the staff focus group during implementation suggest that further communications and consideration of change processes need to be constantly revisited. This suggests that raising awareness and staff engagement should be essential not only at the initiation of the change implementation model, but also at each stage of the process.

While transprofessional practice may be effective, staff are concerned with overlapping roles and have mixed perceptions of the threat to professional identities (Nancarrow, 2004). Previous research suggests that staff seem less threatened with overlapping roles when a collaborative approach is undertaken (Booth and Hewison, 2002), and when staff have confidence in their own role as well as an understanding of other professionals roles (Macdonald et al, 2010; Nancarrow, 2004). In this study, the use of the service and task analysis processes within The Calderdale Framework ensured that staff were aware of their own professional roles in their own team, those of other professionals, as well as the new transprofessional practice.

By completing these processes, clinical staff in this research reflected improvements with professional role knowledge, communication within teams, and client-focused services, while reporting no concerns with role overlap. This is consistent with available literature that recognises that both teamwork and maintaining a client focus (rather than a professional focus) are key aspects of developing transprofessional practices in health settings (Gilmore et al, 2011).

The use of The Calderdale Framework in this community rehabilitation supports the creator's original hypothesis that implementation would lead to increased efficiency of services (Smith and Duffy, 2010). In this study, the resources used for each client appointment decreased, while the number of appointments per client more than doubled from pre-implementation of the framework to post-implementation. By reducing the resources needed for each individual client appointment, staff were then able to offer more client appointments while maintaining overall costs per each client. Thus, clients may have received a more thorough rehabilitation experience, within fixed budget constraints.

Further to this, staff were also able to increase the number of clients seen, suggesting that transprofessional practices help to improve efficiencies of staff time. This may have occurred as the newly trained multi-skilled staff were all able to respond to all initial reviews with clients as soon as time allowed, rather than relying on the availability of different professionals.

\section{Limitations}

This research investigates the use of The Calderdale Framework within a community rehabilitation service to develop a clinically shared transprofessional screening tool. This implementation, however, was narrow in focus and did not explore the use of the Framework in any other aspects of the service beyond the screening tool. It also did not explore delegating suitable screening tasks to therapy assistants to assist with efficiency of service.

\section{Future research}

Further research should be considered to explore the client's views of the transprofessional screening tool, as well as the impacts this may have on functional outcomes. More research would also be beneficial into the use of The Calderdale Framework in developing transprofessional practices for client interventions in Australian community rehabilitation settings.

\section{CONCLUSIONS}

Community rehabilitation is an integral part of Australian health care. However, high service demands requires the need to explore more effective and streamlined health services through flexible workforce development. This research, using The Calderdale Framework, provides further insight of the processes, service outcomes and staff reflections in the community rehabilitation context. This research suggests that this change process is largely positive for staff in improving both client and team focus. Resource usage data suggested that transprofessional practice led to improved service efficiencies.

It is anticipated this research will contribute to the knowledge base in order to provide more effective community rehabilitation services in future demanding health environments. $\quad$ IJTR

Acknowledgements: The authors would like to acknowledge the staff from Queensland Health for their support of this project and research, including the Community Adult Rehabilitation Service team, Metro South Hospital and Health Service District, project sponsor, workforce development officers and the Allied Health Professions' Office of Queensland. 
Funding for this research project was granted by Queensland Health, Allied Health Professions, Office of Queensland.

Declaration of interest: SP and MC were employed by Queensland Health and were responsible for the implementation of The Calderdale Framework as part of a new service model in this community rehabilitation service.

Booth J, Hewison A (2002) Role overlap between occupational therapy and physiotherapy during in-patient stroke rehabilitation: An exploratory study. J Interprof Care 16(1): 31-40

Brusco NK, Taylor NF, Watts JJ, Shields N (2014) Economic evaluation of adult rehabilitation: A systematic review and meta-analysis of randomized controlled trials in a variety of settings. Arch Phys Med Rehabil 95(1): 94-116. doi: 10.1016/j.apmr.2013.03.017

Crotty M, Giles LC, Halbert J, Harding J, Miller M (2008) Home versus day rehabilitation: A randomised controlled trial. Age Ageing 37(6): 628-33. doi: 10.1093/ageing/ afn141

Duckett SJ (2005) Health workforce design for the 21st century. Aust Health Rev 29(2): 201-10

Gilmore LG, Morris J, Murphy K, Grimmer-Somers K, Kumar S (2011) Skills escalator in allied health: A time for reflection and refocus. J Healthc Leadersh 2011(3): 53-8. doi: $10.2147 /$ JHL.S24271

Kendall E, Muenchberger H, Catalano T (2009) The move towards community-based rehabilitation in industrialised countries: Are we equipped for the challenge? Disabil Rehabil 31(26): 2164-73. doi: 10.3109/09638280902939734

Macdonald MB, Bally JM, Ferguson LM, Lee Murray B, Fowler-Kerry SE, Anonson JM (2010) Knowledge of the professional role of others: A key interprofessional competency. Nurse Educ Pract 10(4): 238-42. doi: 10.1016/j. nepr.2009.11.012

Nancarrow S (2004) Dynamic role boundaries in intermediate care services. J Interprof Care 18(2): 141-51

\section{KEY POINTS}

Health care services, including community rehabilitation, need to develop effective streamlined services to meet an increasing service demand

- The Calderdale Framework provides a systematic method of reviewing health services and developing appropriate and timely care via delegation and transprofessional practices

- Staff involved with the creation of transprofessional practices using The Calderdale Framework reported an improved sense of teamwork and client focus

- Greater efficiencies in workforce management and improved client contact following the implementation of transprofessional practices were noted within the context of community rehabilitation services.

Nancarrow S (2015) Six principles to enhance health workforce flexibility. Hum Resour Health 13: 9. doi: 10.1186/1478-4491-13-9

Nancarrow S, Moran A, Wiseman L, Pighills AC, Murphy K (2012) Assessing the implementation process and outcomes of newly introduced assistant roles: A qualitative study to examine the utility of the Calderdale Framework as an appraisal tool. J Multidiscip Healthc 5: 307-17. doi: 10.2147/JMDH.S35493

Queensland Government (2009) Change Management Best Practices Guide. www.psc.qld.gov.au/publications/subject-specific-publications/assets/change-management-bestpractice-guide.pdf (accessed 5 August 2015)

Smith R, Duffy J (2010) Developing a competent and flexible workforce using the Calderdale Framework. Int $J$ Ther Rehabil 17(5): 254-62. doi: 10.12968/ijtr.2010.17.5.47844

Wood AJ, Schuurs SB, Amsters DI (2011) Evaluating new roles for the support workforce in community rehabilitation settings in Queensland. Aust Health Rev 35(1): 86-91. doi: 10.1071/AH10880

\section{Caring for the Person with Dementia}

This book is written with the intention of helping readers understand the perspective of the person with dementia, as well as that of carers and professionals.

It covers:

- Information and debate on theoretical issues and explanations of dementia and memory loss

- Explanations of what dementia is and where it comes from

- The ideas and perspectives of carers and professionals alike

This book will be suitable for nurses, student nurses and carers alike.

ISBN-13: 978-1-85642-308-8; 234 × 156 mm; paperback; 160 pages; publication 2006; £19.99

Order your copies by visiting

or call our Hotline

www.quaybooks.co.uk +44(0)1722716935

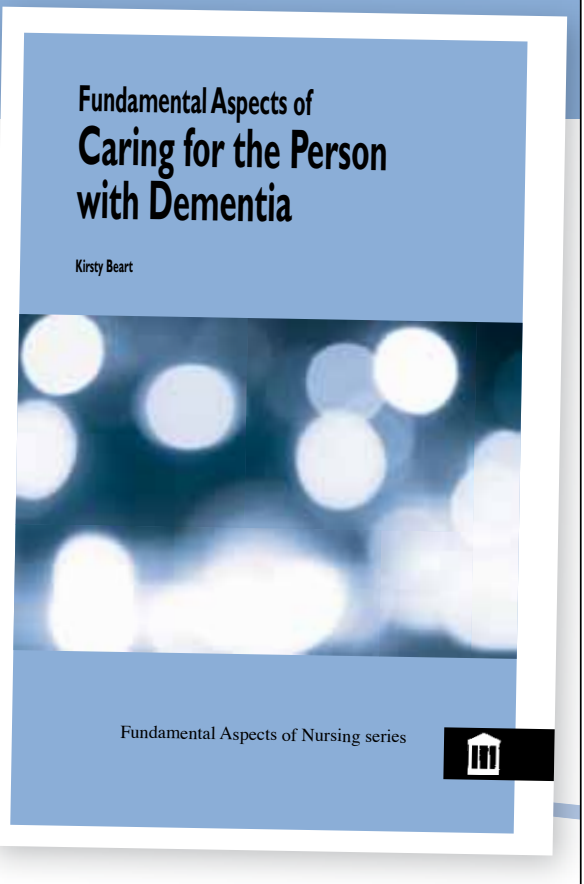


Copyright of International Journal of Therapy \& Rehabilitation is the property of Mark Allen Publishing Ltd and its content may not be copied or emailed to multiple sites or posted to a listserv without the copyright holder's express written permission. However, users may print, download, or email articles for individual use. 